\title{
Reaction time in reading a tachistoscopic display for a memory set item
}

\author{
MARILYN L. SHAW \\ Douglass College, Rutgers- The State University of New Jersey, New Brunswick, New Jersey 08903
}

\begin{abstract}
Reaction times were measured in a task which requires the subject to search a brief visual array for a critical letter embedded in a row of background letters. In Experiment 1, the position of a critical letter in an instructed reading order and the size of a set of memorized letters were varied. Mean reaction time increased monotonically with distance of the critical letter from the beginning of the instructed reading path in the display. The variables reading position and memory set size were additive in their effect on mean reaction time. Data from a second experiment in which the retinal location of the critical letter and its reading position were varied showed that both reading position and retinal location influenced mean reaction time, but the effect of reading position on reaction time was greater. These variables interacted.
\end{abstract}

Sternberg (Note 1) has presented a quantitative algorithm that accurately describes the performance of his subjects and the subjects of Nickerson (1966) in a task that requires a search of a multielement display for a member of a memorized set of letters. This relatively unknown model has considerable appeal because of its simplicity and remarkably good fit to the data from both these experiments. A comparison stage involving two scanning mechanisms is postulated: a scan that is serial, selfterminating in a display image, and a scan that is serial but exhaustive in the memorized set. Testing of a display item involves comparing it successively with every item in the memorized set whether a match is found or not. Items in the display image are tested one at a time until all display items have been compared to the memory set or until comparison to the memory set produces a match. On positive trials, when a match is found, the display scan terminates at the matching display item. In this case, the average number of display items compared to the memory set is $(d+1) / 2$, where $d$ is the number of items in the display. If the size of the memory set is $s$ and $b$ is the time it takes to compare a display item to one memory set item, then on negative trials, when no match is found, the total comparison time is $s * d * b$ and on positive trials the average comparison time is $s *[(d+1) / 2] * b$.

Sternberg found that the six functions relating

This work is based upon a portion of a doctoral dissertation submitted to the University of Illinois. The research was carried out under Public Health Training Grant MH0696 to Ledyard Tucker. The author wishes to thank the following for their helpful comments during various stages of the preparation of this manuscript: Charles Lewis, Charles Eriksen, Saul Sternberg, and Peter Shaw. Requests for reprints should be sent to Marilyn Shaw, Department of Psychology, Douglass College, RutgersThe State University, New Brunswick, New Jersey 08903. mean reaction time $(\overline{\mathrm{RT}})$ to memory set size for each display size ( $d=1,2$, or 3 ) and for positive and negative trials were well fit by straight lines. The entire set of data was simultaneously fitted to linear functions under the constraint that the estimated mean comparison time is the same for all of them, and this one parameter model provided an unusually good fit. In spite of the fact that displays in Sternberg's experiment were briefly exposed while displays in Nickerson's experiment were exposed until the subject responded, the estimated value of the comparison time parameter was about the same for both sets of data.

A basic assumption of Sternberg's model is that changes in RT with display size reflect only changes in the number of comparisons in his comparison stage. An alternative possibility considered by Sternberg (Note 1) is that the variable display size may also affect the duration of an input stage in which images of the display items are formed. Sternberg argued that if display size does affect the duration of an input stage, then the intercept of each function relating $\overline{\mathrm{RT}}$ to memory set size should vary with display size. However, Sternberg found no systematic variation in the intercepts of these functions with display size in either his or Nickerson's data, and from this he inferred that display size affects only the number of comparisons in the comparison stage.

The observation that display size may affect the duration of more than one information processing operation is an important one, and Sternberg's inference that this is not the case is plausible given the assumptions of his model. However, it would be desirable to investigate this model using a variable that is not likely to affect the time it takes to form an image of the display. A variable used by Shaw (1969) and Shaw and LaBerge (1971) seems promising in 
this regard. In these studies, display size was fixed and the position of a critical letter was varied. In Shaw (1969), each trial began with a subject's eyes fixated on a dot. Next, a horizontal linear array of letters was briefly presented to the right of the focusing dot. The subject's task was to "read" the letters from left to right and to indicate which of two alternative critical letters appeared somewhere in the display. Each display contained one critical letter, and the other letters were background letters, either an $\mathrm{N}$ or $\mathrm{H}$, irrelevant to a determination of the correct response. Shaw (1969) and Shaw and LaBerge (1971) found that reading position has a large effect on performance accuracy: the earlier the reading position, the more accurately the critical letter was recognized. Shaw and LaBerge (1971) interpreted these in terms of a preserving operation that successively processes items in the display image according to the instructed reading order. The preserving operation on each item, they speculated, might be the comparison operation that Sternberg had postulated for the character classification task with a single letter display. For the forced-choice recognition paradigm they used, this means that each display item is compared to the two alternative critical letters and that the order in which display items are compared to these letters can be controlled by the experimenter's instructions to the subject.

There are two reasons why the Shaw and LaBerge (1971) conjecture about the generality of Sternberg's (Note 1) algorithm cannot be tested with their data: they did not vary the number of memory items that must be compared to display items, and their dependent measure was performance accuracy and Sternberg's model is formulated for reaction time.

The present investigation was motivated by consideration of the advantage of using the variable reading position instead of display size for investigation of Sternberg's algorithm and by an interest in testing the generality of this algorithm as suggested by Shaw and LaBerge (1971). For these purposes, the paradigm of Experiment 1 was designed so that display size was fixed, reading position was varied, several memory set sizes were used, and the dependent measure was RT.

Sternberg's algorithm (Note 1) can be modified for the variable reading position in a natural way by assuming that, on positive trials, reading position rather than display size determines the number of comparisons of display items to the memory set. Items in the display image are tested one at a time until all display items have been compared to the memory set or until a comparison to the memory set produces a match. If the rearing position of an item in the display is $p$ and the size of the memory set is $s$, then the total number of comparisons is $s * p$ and the total comparison time is $s^{*} p^{*} b$, where $b$ is the time to compare one display item to one memory set item. On negative trials, all items in the display are compared to the memory set and the total comparison time is $s^{*} d^{*} b$, where $d$ is the number of items in the display. The data from the present experiment will be adequately described by this version of the comparison process if the slope of the function relating RT and memory set size, with reading position as a parameter, is directly proportional to reading position of a positive item and if there is an interaction between reading position and memory set size, between reading position and response type, and between memory set size and response type.

In the displays of Shaw (1969) and Shaw and LaBerge (1971), in contrast to those of Sternberg (Note 1), there are redundant background letters irrelevant to the determination of a correct response, besides a critical letter. Results from a number of studies (Estes, 1974; McLaughlin, Masterson, \& Herrmann, 1972; McIntyre, Fox, \& Neale, 1970) have shown that identification of a critical letter is faster and more accurate for displays with redundant background letters than for displays such as those used by Sternberg (Note 1). These results suggest that the presence of redundant background letters affects the information processing requirements of the task. It may be that the number of comparisons of display items to critical items is reduced in this case.

The possibility that displays with redundant background letters may require fewer comparisons to the memory set suggests an interesting variation of the above serial algorithm for reading position that makes quite different predictions for the effect of reading position and memory set size on RT. Suppose each display item is subjected to a preliminary test for membership in the set of background letters, then, for a given location, a negative outcome of this preliminary test means that the item in that location is a critical letter. The critical letter is then compared exhaustively to the memory set and no further items in the display are scanned. The total number of preliminary tests is independent of memory set size, but is a function of the reading position of the critical letter, while the total number of comparisons to the memory set is a function of the set size, but is not a function of reading position. In this case, the effect of the variables reading position, memory set size, and response type should have additive effects on $\overline{R T}$.

\section{EXPERIMENT 1}

The purpose of Experiment 1 was to determine the shape of the $\mathrm{RT}$ vs reading position functions and to determine whether the slope of the function relating $\mathrm{RT}$ to memory set size with reading position as a parameter depended upon the size of the memory set or upon both memory set size and reading position. To provide a strong test of the general- 
ity of Sternberg's (Note 1) algorithm and to test the Shaw and LaBerge (1971) hypothesis that all display items in their task were compared to the set of critical letters, it was decided to use displays similar to those of Shaw (1969): a single critical letter was presented in a horizontal linear array of redundant background letters. The critical letter was either a member of a set of positive, memorized letters or a member of a set of negative letters, and for a block of trials the number of letters in each of these sets was the same. The number of letters in a display was fixed and reading position of the critical letter was varied.

\section{Method}

Subjects. Four University of Illinois undergraduate students, one female and three males, served as subjects and were paid for their participation in the experiment. All subjects had $20 / 20$ uncorrected vision and were right-handed.

Apparatus. The experiment was conducted in a soundproof, air-conditioned room in which the experimenter, subject, and apparatus were located. A Scientific Prototype Model GB Auto Tachistoscope was used to present the visual stimuli. The stimulus field and the adaptation field had a luminance of $14.733 \mathrm{~cd} / \mathrm{m}^{2}$, as measured by a Spectra Spot Photometer.

Reaction times were measured using a General Radio Model 1192-B counter in conjunction with a megacycle pulse generator. Subjects initiated the counter and stimulus presentation by depressing a foot pedal and stopped the counter by pressing a lever located on their right side. The lever was pressed left or right depending on the subject's response selection.

Stimulus materials. There were 96 different visual displays used in this experiment. The displays were made by photographing white letters on a black background with $35-\mathrm{mm}$ highcontrast copy film and then mounting the negatives as slides. The letters, 30-pt capital News Gothic reversed, were made by a Varitype Headliner and were $.0076 \mathrm{~m}$ high and $.0047 \mathrm{~m}$ wide with a space between them. The slides were seen as black letters on a bright background. Each display contained a horizontal linear array of 10 letters subtending approximately $2^{\circ}$ of visual angle, and the first six letters subtended approximately $1^{\circ} 19^{\prime}$ of visual angle.

Each 10-letter display had one critical letter and nine background letters. The critical letter was either from the negative set of letters or the positive set of letters memorized by the subject, and the letters for these sets were selected from the set of letters B, D, F, H, L, J, P, R, S, T, Z, V. The critical letter appeared in the first, second, fourth, or sixth reading position in the array (reading position is computed from left to right). For each reading position, there were two different backgrounds. The first was obtained by randomly selecting a background letter $(\mathrm{N}$ or $\mathrm{X})$ for each of the display positions, with the constraint that runs of a given background letter be no longer than three and that the critical letter position be flanked by the same background letter on the left and right. The second background was obtained by replacing $N s$ with $X s$ and $X s$ with $N s$ in the first background.

Fixation was provided by two vertical bars positioned so that one was directly above and the other directly below the leftmost letter position of the display. Each bar was one letter in height, the two being separated by a space equal to the height of one and one-half letters. This type of fixation was used so that any possible spatial or temporal interaction between the letter in the first position and the fixation markers would be minimized. Subjects were instructed before each block of trials to focus on the space between the vertical bars before pressing the foot pedal.

Procedure. The subjects participated in 4 practice sessions and 19 sessions of data collection. Each session lasted about $40 \mathrm{~min}$, and the sessions were divided into four blocks of 32 trials.
In each session, the subject memorized four different sets of letters containing two, three, four, or five letters selected from the set of 12 stimulus letters. The subject was instructed to press the lever to the right if the critical letter in the display was a member of the memory set and to the left if it was not. In order to equate for possible stimulus letter probability effects in a block of trials, the number of critical letters in the negative set was the same as the number in the positive, memorized set. At the beginning of a block of trials, the subject was told to memorize the set of letters (positive set only) in the order given to him by the experimenter and to repeat out loud the memory set immediately after each response. At the beginning of each block, the subject was told what the negative critical letters were, but that it was not necessary to memorize them. Prior to each block of trials, the subject was given practice trials in which each of the possible critical letters for that block of trials was presented once. The subjects were told that the critical letter would appear in only the first, second, fourth, or sixth display position.

During the first and second practice sessions, the display duration was decreased from $200 \mathrm{msec}$ to a final duration of $100 \mathrm{msec}$. At the beginning of each block of trials, the subject was reminded that he should fixate between the bars before initiating a trial. The subjects were instructed to be as fast as possible, but to be at least $95 \%$ correct in responding. Feedback on errors was given during practice, but not during data collection.

The subjects were dark-adapted for $5 \mathrm{~min}$ at the beginning of each session and were allowed to rest a few minutes between blocks of trials.

Within a session, each stimulus letter occurred at least once as a member of a positive memory set and once as a member of a negative set. The following variables were balanced within a block of trials: frequency of positive and negative trials, frequency of a critical letter in a reading position, and frequency of a background type. For each session, a subject received the memory set sizes in one of the 24 possible orders, randomly selected without repetition. The serial position of a letter in a memory set was selected at random, with the constraint that over all sessions each letter on the average occur equally often in each possible serial position for a given memory set size. Trial numbers were assigned randomly to stimuli in a block, with the constraint that a run of positive or a run of negative trials be no longer than five.

Payoffs. Payoffs were designed to encourage fast, accurate responses and compliance with the instructions to fixate. Errors on targets in the second, fourth, and sixth reading positions were penalized 10 points. Errors on targets in the first reading position were penalized 20 points. A point was gained for every $10 \mathrm{msec}$ the average reaction time to critical letters in a block was less than a minimum time determined from practice session data. The subjects were told they could earn from $\$ 1.50$ to $\$ 2.50$ per session; that the subject with the greatest number of accumulated points over all data collection sessions would receive $\$ 2.50$ per session; and that other subjects would be paid according to their performance relative to the subject with the greatest number of points accumulated.

Every fourth session, the subjects were told how many points they had accumulated and how many points the other subjects had accumulated. They were also told how many errors they had made over the four sessions.

\section{Results}

Summary statistics computed for each session and for groups of four successive sessions showed that, until Session 9, $\overline{\mathrm{RT}}$ continued to decrease. To avoid including data subject to practice effects, only the data collected in Sessions 9-23 were analyzed. Error latencies were excluded from all analyses of reaction time. Error rates for Reading Positions 1, 2, and 4 
were below 5\%, and error rates for Reading Position 6 ranged from $4 \%$ to $26 \%$.

Mean reaction time as a function of memory set size with reading position as a parameter is graphed separately for each subject in Figure 1. Mean reaction time as a function of reading position with memory set size as a parameter is graphed separately for each subject in Figure 2. For each subject, $\overline{\mathrm{RT}}$ increases monotonically with increases in memory set size and reading position.

For each combination of subject and response type presented in Figure 1, parallel lines were simultaneously fitted with a least squares procedure to the graphs relating RT to memory set size for each reading position. The slopes of these parallel lines for positive and negative responses are approximately the same, while the intercepts of these functions are greater for negative responses than for positive responses. The estimated proportions of variance accounted for by the best fitting parallel lines for each subject and response type are presented in Table 1. The estimated slopes and intercepts of these lines are presented in Table 2 . The extremely good fit of the parallel linear functions indicates that memory set size, reading position, and response type are additive factors. The proportions of variance accounted for by the additive model were 96 , $.91, .98$, and .97 for Subjects 1, 2, 3, and 4, respectively.

Inspection of the graphs in Figure 2 shows that, for three of the four subjects, the function relating $\overline{\mathrm{RT}}$ to reading position is nonlinear. An F test showed that the deviations from linearity for the data of Subjects 1, 2, and 4 were significant. For these subjects, the difference in $\overline{\mathrm{RT}}$ for Reading Positions 1 and 2 are extremely small and $\overline{R T}$ for Reading Position 6 is extremely long. Since eye fixations were not monitored, the small difference in $\overline{\mathrm{RT}}$ for the first two reading positions may reflect fixation inaccuracy.

Table 1

Proportion of Variance Accounted for by Prediction from Simultaneously Fitted Parallel Lines*

\begin{tabular}{ccccc} 
& \multicolumn{4}{c}{ Subject } \\
\cline { 2 - 5 } Responses & 1 & 2 & 3 & 4 \\
\hline Positive & .99 & .97 & .99 & .99 \\
Negative & .96 & .92 & .99 & .97 \\
\hline
\end{tabular}

* Reaction time as a function of memory set size.

Note-W is $1-A / B$, where

$$
A=n \sum_{j k}\left(\bar{Y}_{\cdot j k}-Y_{j k}\right)^{2}
$$

and

$$
B=n \Sigma \Sigma\left(\bar{Y}_{\cdot j k}-\bar{Y} \cdots\right)^{2},
$$

where $\hat{Y}_{j k}$ is the mean predicted by the best fitting parallel lines for the $j^{\text {th }}$ target position and the $k^{\text {th }}$ set size. $\bar{Y}$ is the actual mean for that condition and $\bar{Y} \cdots$ is the grand mean.
Table 2

Slopes and Intercepts of the Simultaneously Fitted Parallel Lines for the Function Relating Reaction Time and Memory Set Size for Each Reading Position

\begin{tabular}{rlrrrrr}
\hline & & \multicolumn{4}{c}{ Intercepts } \\
\cline { 3 - 6 } Subject & \begin{tabular}{c} 
Response \\
\cline { 3 - 6 }
\end{tabular} & Type & \multicolumn{4}{c}{ Reading Positions } \\
\cline { 3 - 6 } & 1 & 2 & 4 & 6 & Slope \\
\hline \multirow{2}{*}{1} & Positive & 403 & 414 & 452 & 581 & 38.39 \\
& Negative & 489 & 481 & 564 & 712 & 33.65 \\
2 & Positive & 430 & 437 & 477 & 557 & 23.13 \\
& Negative & 508 & 505 & 582 & 739 & 23.62 \\
3 & Positive & 541 & 637 & 849 & 992 & 20.67 \\
& Negative & 574 & 654 & 894 & 1022 & 21.48 \\
4 & Positive & 395 & 410 & 512 & 773 & 32.06 \\
& Negative & 481 & 483 & 560 & 789 & 27.14 \\
\hline
\end{tabular}

To summarize, the important result from Experiment 1 is the additivity of reading position, memory set size, and response type.

\section{Discussion}

The paradigm of Experiment 1 combined two tasks: the examination of a brief display along an instructed reading path for a relevant critical letter and the comparison of a critical letter to a memorized set of letters. Despite the unusual test stimulus for presentation of the critical letter, Experiment 1 produced the typical finding from the character classification paradigm: RT was a linear function increasing with memory set size, and these functions were parallel for positive and negative responses. The memory comparison rate, $b$, estimated from the present data, varies with the subject and ranges from 20 to $38 \mathrm{msec}$, but the values are consistent with values estimated in a number of other studies.

The serial algorithm for reading position which has each display item compared to the memory set predicts an interaction between reading position, memory set size, and response type. The additivity of these variables in the data of Experiment 1 is not consistent with this, but these results can be accounted for by the second version of this model which has, instead, a preliminary test of display items for membership in the set of background letters and only the critical letter is compared to the memory set. According to this algorithm, the time it takes for the display scan to reach the critical letter in the display image is a function of its reading position and not a function of the size of the memorized set. Conversely, the time it takes to compare the critical letter to the memory set is a function of the set size and not a function of its reading position.

Evidence for the importance of discrimination of background letters from critical letters in Experiment 1 comes from examination of error rates and reaction times for the individual critical letters. In terms of vertical and diagonal lines, the letters $H, R$, and $V$ share features with the background letters $\mathrm{N}$ and $\mathrm{X}$ that are not shared to the same extent with 
Figure 1. The functions relating $\overline{\mathrm{RT}}$ to memory set size for each reading position is graphed separately for each subject and response type.

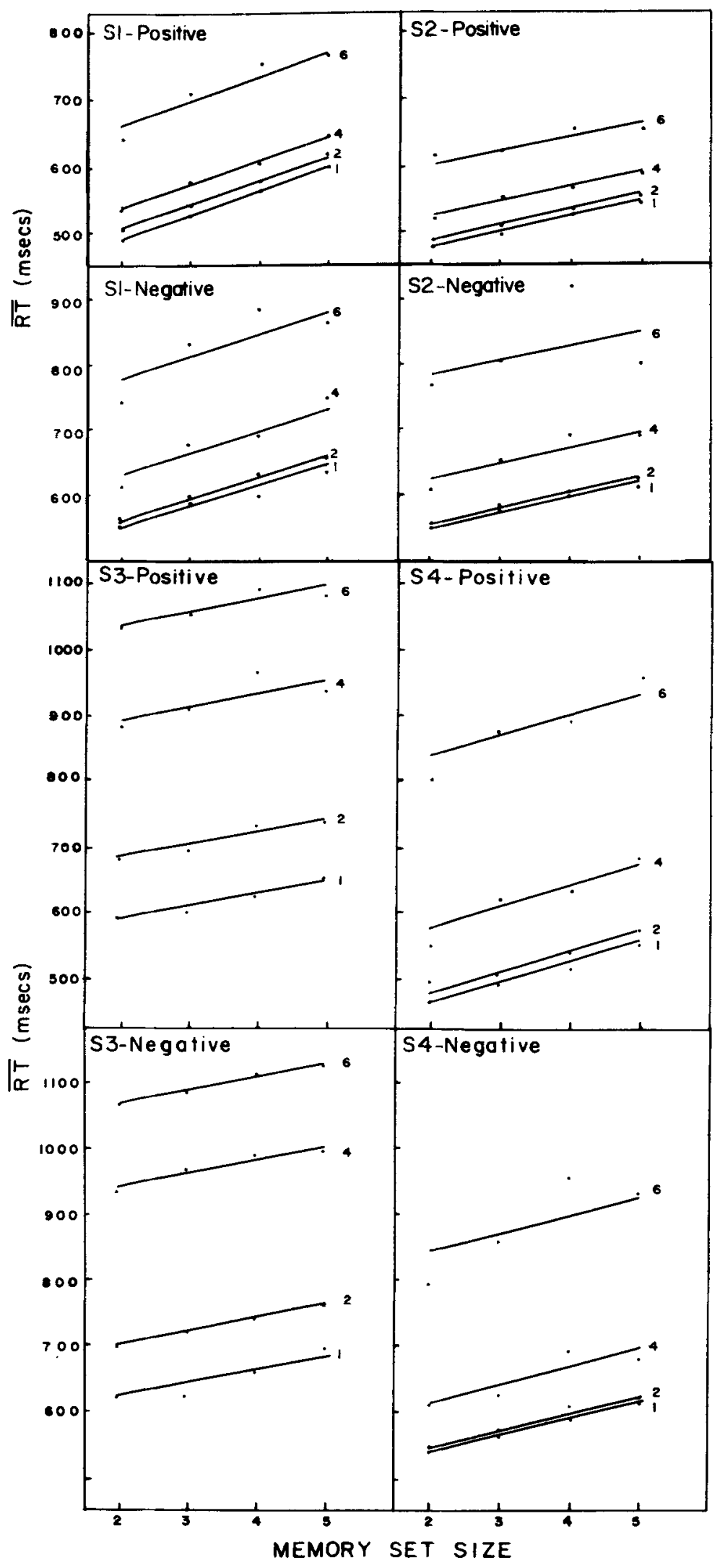




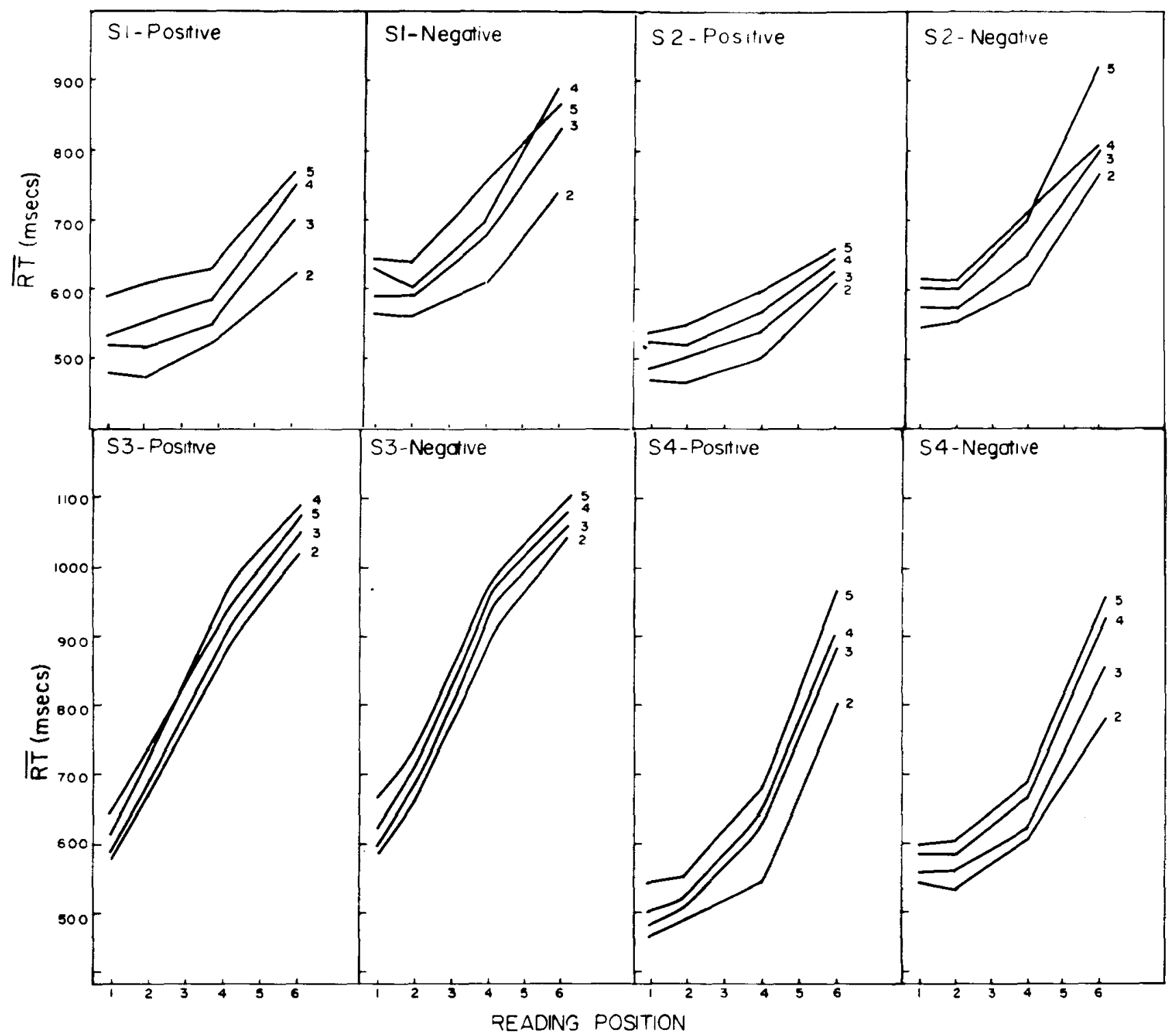

Figure 2. The functions relating $\overline{\mathrm{RT}}$ to reading position for each memory set size is graphed separately for each subject and response type.

the other critical letters. The average error rate for the letters $H, R$, and $V$ were between $11 \%$ and $13 \%$, while for the letters B, D, F, J, L, P, S, T, and Z it was under $5 \%$. For each subject, graphs of $\overline{\mathrm{RT}}$ vs reading position for the individual letters showed that $\overline{R T}$ to the letters $H, R$, and $V$ were always longer than $\overline{R T}$ to the other letters. The smallest average difference was $30 \mathrm{msec}$ for Reading Position 1, and the largest average difference was $220 \mathrm{msec}$ for Reading Position 6.

The nonlinearity of the reading position functions, together with the error and RT data for individual letters, suggests that the time taken by a mechanism for discriminating between background and critical letters is not constant for all reading positions. There are several ways to interpret the nonlinearity of these functions: (1) The image of the display is rapidly decaying so that, over time, information in locations late in the reading path is highly degraded by the time the scanning mechanism reaches them; (2) the quality of the initial image on the retina decreases with distance of reading position from fixation, even before the decay process begins; and (3) the rate of information extraction from display image locations decreases with distance from fixation. Though the possibility of simultaneous processing of display locations has not been considered in the present discussion, it is clear that interpretations 2 and 3 could account for the entire effect of reading position on RT. The confounding of reading position and retinal 
location in Experiment 1 makes it impossible to determine the contribution of retinal image quality to the reading position functions of this experiment.

\section{EXPERIMENT 2}

Evidence against an interpretation of the effects of reading position on performance accuracy solely in terms of the quality of the initial retinal image has been presented by Estes and Wolford (1971) and Shaw and LaBerge (1971). Shaw and LaBerge constructed their visual displays so that each reading position was equidistant from the point of fixation, and found an effect of reading position similar to that of Shaw (1969). Estes and Wolford independently varied retinal location and reading position in a full report paradigm, and found that reading position had a larger effect on report accuracy than retinal location and that these two variables interacted. Though these results do not favor a retinal location interpretation of the data of Experiment 1, the differences in paradigms and dependent measures make generalization to the present data difficult.

In Experiment 2, the design of Estes and Wolford (1971), varying the location of the display in the visual field, was used to assess the relative contributions of reading position and retinal location on RT and to determine whether their effect on RT was additive or interactive.

\section{Method}

Four University of Illinois students served as paid subjects. All subjects were naive and right-handed, and had uncorrected $20 / 20$ vision.

Apparatus. The equipment and experimental room used were the same as in Experiment 1. The stimulus and adaptation field had a luminance of $14.733 \mathrm{~cd} / \mathrm{m}^{2}$, as measured by a Spectra Spot Photometer.

Stimulus materials. Stimuli were made using the procedures of Experiment 1. The visual angle subtended by the 10 letters was the same as in Experiment 1. The critical letter was chosen from the set of letters $D, L, J, P, S$, and $Z$.

The experimental factors were reading position of the critical letter, retinal location of the critical letter, and response type (yes, no). There were three reading positions $(1,4,7)$ and five retinal locations numbered from -6 through 0 to +6 , with location 0 referring to the center of the field or fixation. Negative and positive numbers for retinal location refer to locations in the left and right visual fields, respectively. The five retinal locations selected for presentation of the critical letter were $-6,-3$, $0,+3,+6$. Reading Position 1 appeared in Retinal Locations -6 , $-3,0$, and +3 ; Reading Position 4 appeared in Retinal Locations $-3,0$, and +3 ; and Reading Position 7 appeared in Retinal Locations $0,+3$, and +6 . The distance from Retinal Locations -6 and +6 subtended a visual angle of $1^{\circ} 24^{\prime}$, and the distance from Retinal Locations -3 and +3 subtended a visual angle of $42^{\prime}$. This design is unbalanced because the size of the viewing field in the tachistoscope made it impossible to combine each of the selected reading positions with all of the selected retinal locations used in the experiment. Fixation bars and background letters were the same as in Experiment 1. The leftmost letter of the display was called the verification letter and was $N, X$, or one of the six possible critical letters. If a verification letter was one of the critical letters, it also served as a critical letter in Reading Position 1.
Procedure. The task of Experiment 2 was the same as in Experiment 1 except that subjects were required to report the leftmost letter of the display as a verification letter. During the first two practice sessions, subjects were not required to report the leftmost letter of the display, and they reported having great difficulty reading the displays from left to right when the display appeared in the left visual field. Their inability to follow the instructions to "read" the display from left to right was confirmed by the high error rates on critical letters in the first reading position when the display appeared in the left visual field. An instruction to report the leftmost letter (verification letter) in the display was incorporated into the subject's task and eliminated this difficulty. Report of a verification letter was used by Shaw (1969) and Shaw and LaBerge (1971) to insure accuracy of fixation.

For each block of trials, the subject memorized a set of three letters. There were three practice trials at the beginning of each block, and the subject was reminded of the instruction to fixate between the bars before depressing the foot pedal and to read the display from left to right, regardless of the display's location in the visual field. On each trial, the display was illuminated for $150 \mathrm{msec}$. In all other respects, the procedures of this experiment were the same as in Experiment 1.

Payoffs. The subjects were instructed to be at least $95 \%$ accurate in responding to critical letters and nearly $100 \%$ accurate in reporting the verification letter. Penalty points were given for errors as follows: (1) 30 points for an error in reporting the verification error, (2) 20 points for an error in responding to a critical letter in the first reading position, and (3) 10 points for all other errors. Subjects were informed of this point system at the beginning of the experiment and were told that at the end of the experiment, subjects would be ordered according to their accumulation of points. The subject with the greatest number of points would receive $\$ 2.00$ per session. The other subjects would receive $\$ 2.25, \$ 2.50$, and $\$ 2.75$ per session, according to the number of points accumulated. In addition, a $\$ .10$-persession bonus was awarded to the subject with the fastest $\overline{\mathbf{R T}}$.

\section{Results}

Mean reaction time averaged over the four subjects in Experiment 2 as a function of retinal location for each of the three reading positions is presented in Figure 3. The graph of the data averaged over subjects is representative of the graph of each individual

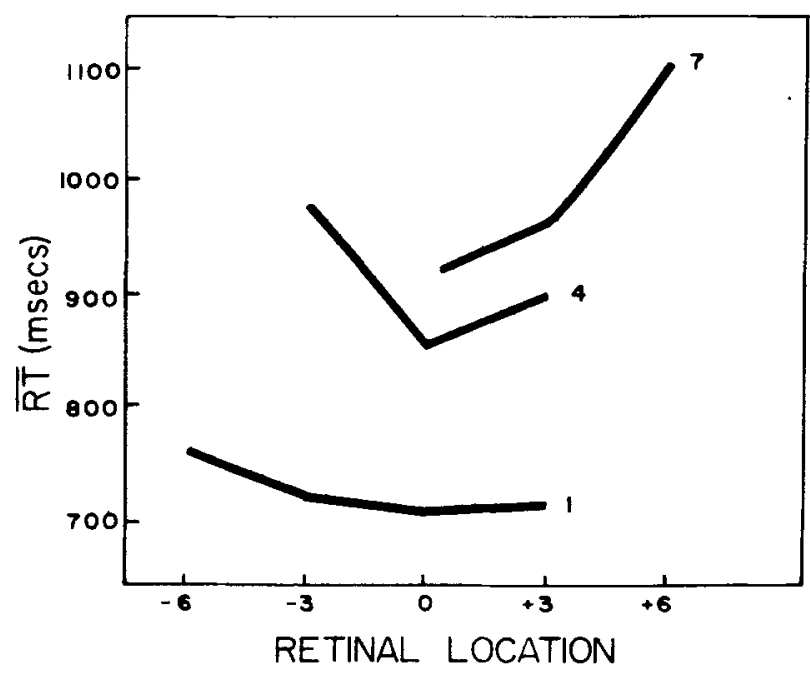

Figure 3. Mean reaction as a function of retinal location, with reading position as a parameter averaged over the four subjects in Experiment 2. 
Table 3

Summary Statistics

Experiment 2

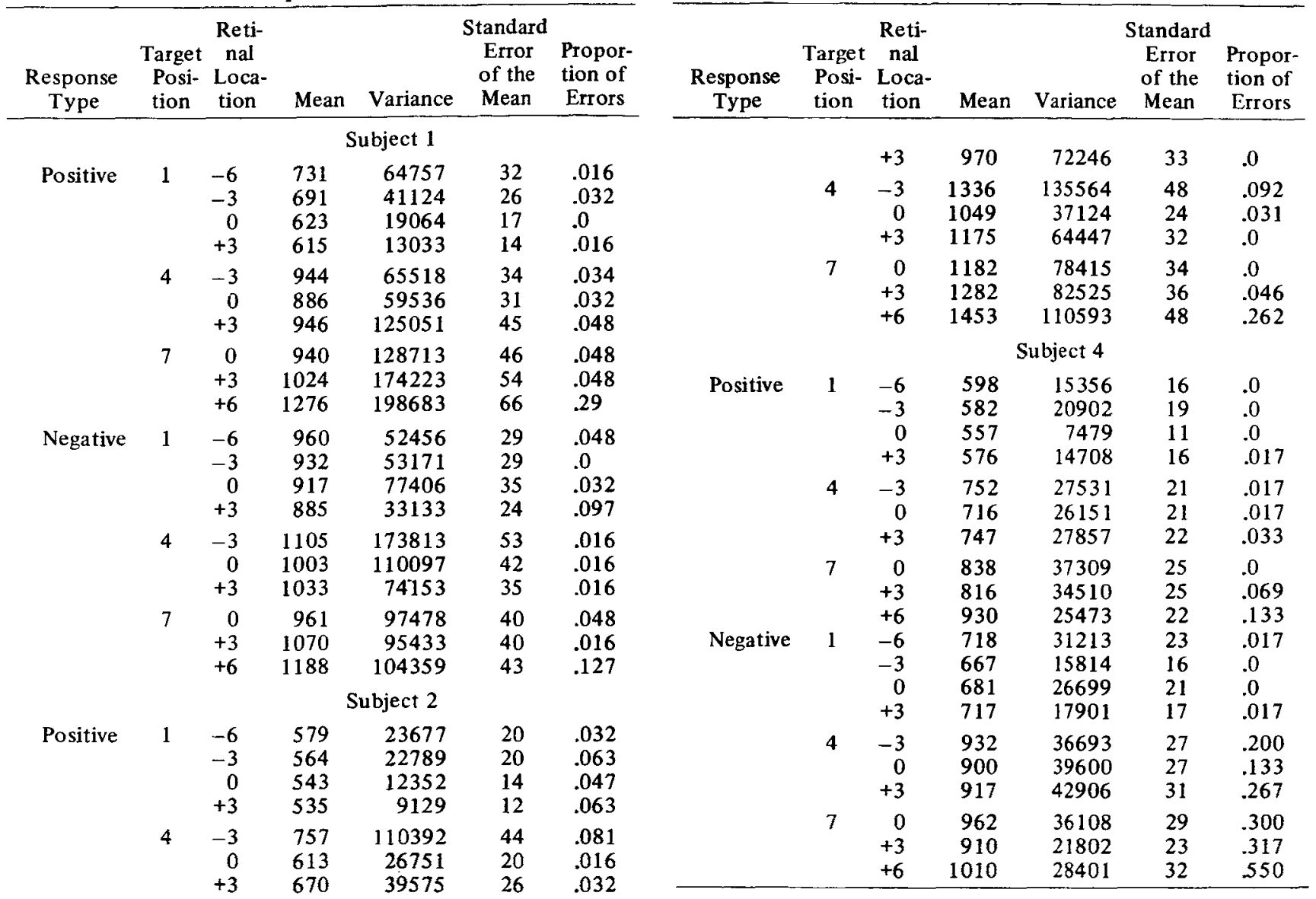

subject's data. For $\overline{\mathrm{RT}}$ to critical letters in Reading Position 1, retinal location appears to have a minimal effect in contrast to $\overline{\mathrm{RT}}$ for Reading Position 7.

Table 3 summarizes the data for individual subjects in this experiment.

The analysis of Experiment 2's data presented several problems. First, the design is unbalanced and nonorthogonal. Second, the assumption of homogeneity of variances required by the $F$ test was violated in $90 \%$ of the analyses planned (F max test, Winer, 1971). Third, inspection of the RT distributions showed that in every case the distributions were positively skewed with large right hand tails. Fourth, the variance is a monotonic increasing function of the mean. It was concluded that the validity of using the $F$ test with this data was questionable. However, the analysis of variance computations provide the statistics necessary to estimate the proportion of variance accounted for by the experimental variables. The interpretation of this proportion does not require any distribution assumptions, and since the primary interest of Experiment 2 was to assess the relative contribution of the experimental variables to $\overline{\mathrm{RT}}$, it was decided to use this measure of statistical 
association. The proportion for a given effect is the ratio of the sum of squares for the effect to the total sum of squares for all effects.

Because the design was unbalanced, all experimental conditions were not analyzed simultaneously. Submatrices of the design were selected so that the proportion of variance accounted for by each of the experimental variables could be examined for subsets of the experimental conditions.

The effect of reading position, retinal location, and response type was analyzed for the Retinal Locations $-3,0$, and +3 and Reading Positions 1 and 4 . This analysis shows the relative contribution of these variables within the middle range of their values in Experiment 2. For each subject, reading position accounted for the largest proportion of variance $(.5, .7, .7$, and .6 for Subjects $1,2,3$, and 4 , respectively). Retinal location never accounted for more than $17 \%$ of the variance. Thus, within a visual angle of $42^{\prime}$, the effect of retinal location is small relative to the effect of reading position. Examination of Table 3 for these combinations of retinal location and reading position confirms the outcome of this analysis. Looking at Figure 3 , it is seen that retinal location has a definite effect on $\overline{\mathrm{RT}}$ to critical letters in Reading Position 4 , whereas it has a very minor effect on $\overline{\mathrm{RT}}$ to critical letters in Reading Position 1.

All reading positions occurred in Retinal Locations 0 and +3 , making it possible to look at the effect of all reading positions simultaneously for these two retinal locations. Again, position of the critical letter in the instructed reading order accounted for a large proportion of the variance $(.6, .9, .7$, and .7 for Subjects 1, 2, 3, and 4, respectively). Retinal location of the critical letter accounted for no more than $7 \%$ of the variance in any subject's data. The remaining effect variance in this analysis is attributable to response type.

To examine the effect of retinal location and response type for each reading position, a separate analysis was done for all the retinal locations occurring with a given reading position. Table 4 summarizes the results of this analysis for each reading position. Inspection of the table shows that, in general, the effect of retinal location on RT increases with increases in reading position and that these two variables interact.

Inspection of the proportion of response errors for each subject and experimental condition indicated that subjects were, for the most part, at least $95 \%$ correct in responding. The notable exception to this is for critical letters occurring in Reading Position 7 and Retinal Location +6 , and for this condition error rates ranged from $6 \%$ to $55 \%$. An examination of the proportions of verification errors for each subject showed that in no case was the proportion greater than .03 .
Table 4

Proportion of Variance Accounted for by Retinal Location and Response Type for Reading Positions 1, 4, and 7 in Experiment 2

\begin{tabular}{clccc}
\hline & & \multicolumn{3}{c}{ Reading Position } \\
\cline { 3 - 5 } Subject & & 1 & 4 & 7 \\
\hline \multirow{2}{*}{1} & Retinal Location & .08 & .22 & .94 \\
\multirow{2}{*}{2} & Response Type & .92 & .74 & .001 \\
& Retinal Location & .76 & .84 & .69 \\
3 & Response Type & .20 & .15 & .25 \\
& Retinal Location & .20 & .71 & .75 \\
4 & Response Type & .72 & .21 & .22 \\
& Retinal Location & .07 & .03 & .44 \\
& Response Type & .91 & .97 & .55 \\
\hline
\end{tabular}

\section{Discussion}

Experiment 2 was concerned with the relative contributions of retinal location and reading position to the increase in $\overline{\mathrm{RT}}$ with distance of the critical letter from the beginning of the instructed reading order in Experiment 1. For each subject, reading position accounted for a major proportion of variance. An effect of retinal location on the time to respond to a critical letter is also present in the data of Experiment 2. The figures and analyses indicate that as reading position increases, the contribution of retinal location to $\overline{\mathrm{RT}}$ increases. Thus, even when the entire display image lies within the fovea, there is an interaction between reading position and retinal location. This suggests that the stage of processing influenced by reading position is early enough in the sequence of processing stages to be influenced by the sensory variable of retinal location (Sternberg, 1974, Note 2).

\section{GENERAL DISCUSSION}

Serial scanning of a fading trace has been widely used as an explanation for the effects of variations in display size on latency and accuracy. From this viewpoint, the reading position of a critical letter and display size influence the same processing operations, but reading position differs from display size in that it is not likely also to influence initial formation of the display image. The large monotonic increase in $\overline{R T}$ with reading position observed in the present experiments shows that this variable is a viable alternative to display size for investigation of visual information processing. The data of Experiment 2 demonstrate that not all of the effect of reading position on RT can be accounted for by a gradient of retinal sensitivity corresponding to the reading positions used in Experiments 1 and 2.

If it is assumed that every display item is successively compared to the memory set until a match occurs or all display items have been 
compared, then there should be an interaction between reading position and memory set size. In the present investigation, this model was tested for displays in which a critical letter was embedded in a row of background letters and the outcome of this test was negative. The additivity of reading position and memory set size in the data of Experiment 1 suggests that a preliminary test was performed in which display items were tested for membership in the set of background letters and that only the critical letter was compared to the memory set. In addition, the data for individual letters suggests that this test may be based upon an analysis of features. With the present set of data, it is difficult to specify further the nature of such a preliminary test as there was not systematic variation of factors that might influence the duration of the test and whether it is appropriate for the task. If the experimenter changed the background set every few trials, for example, it is unlikely that the subject's information processing procedure would be built around a preliminary test of each display item for membership in the background set.

Serial scanning models have proven amenable to quantitative formulation and have given good fits to some of the data from visual search tasks, but the view that processing of display image locations is simultaneous is equally defendable (cf. Eriksen \& Spencer, 1969; Estes, 1972; Gardner, 1973a; Rumelhart, 1970), though specific mathematical descriptions are frequently equivalent to a serial model (Townsend, 1971). One view of simultaneous processing of display image locations, presented by Eriksen and Spencer (1969) and Gardner (1973a), assigns the effect of display size on performance accuracy to a later decision stage responsible for organization of perceptual information for response processing. Gardner $((1973 b)$ has given a specific interpretation of the effect of reading position in the accuracy data of Shaw (1969) and Shaw and LaBerge (1971) in terms of a function that weights the relative importance of display items during response processing. This weighting function corresponds to the instructed reading order and, in the case of Shaw and LaBerge (1971), the payoff associated with each reading position. At present, there are several limitations on Gardner's interpretation. First, it fails to account for an asymmetry found by Shaw (1969) and others (Estes \& Wolford, 1971; Townsend, Taylor, \& Brown, 1971; Wolford \& Hollingsworth, 1974a, b) in the effect of a space or solid bar adjacent to a critical letter; second, this model has not been developed to the point of making detailed predictions about latency data; and third, a mathematical description of the function postulated by Gardner remains unspecified.

Another view of simultaneous display processing assigns the effect of display size on performance to early "perceptual" processing. Estes (1972) has formulated a qualitative model of this type that takes into consideration a wide variety of variables known to affect performance in visual search tasks. Of particular interest for the present study is the use of interactions between elements in the display image to explain serial position effects in full report data. In a recent study, Estes, Allmeyer, and Reder (1976) varied retinal position in both the left and right visual fields of a four-letter linear display flanked by masking characters ( $\$$ or \#). The serial position functions showing report accuracy vs item position in the display were U-shaped, with the leftmost (first) and rightmost (last) letters being most accurately reported. For the first and last serial positions, relative distance of the letter from fixation depended upon the visual field used for display presentation, and for these two positions the one closest to fixation was more accurately reported. Estes et al. (1976) suggest that these results can be accounted for by two assumptions: a subject's information about the serial position of an item in the display decreases with distance of that item from fixation, and there is mutual interference of neural responses to characters imaged on adjacent retinal locations. With respect to lateral interference of adjacent characters, it is further assumed that these interference effects increase with distance from fixation, are greater on the peripheral side of a letter than on the central side, and are greater for physically similar characters than for physically dissimilar characters. In principle, the interaction between reading position and retinal location-especially the difference between $\overline{\mathrm{RT}}$ function for Reading Position 1 and the other reading positions-in the data of Experiment 2 and the effect of feature similarity between critical and background letters on $\overline{R T}$ and accuracy in Experiment 1 can be accounted for in terms of these assumptions for lateral interference of adjacent characters. However, if these assumptions are to account for the entire effect of reading position in the present experiments, then in the data of Experiment 2 there should be no difference in $\overline{R T}$ for two reading positions imaged at the same retinal location and surrounded by the same background letters. In Experiment 2, Reading Positions 4 and 7 were presented at Retinal Location 0 and also at Retinal Location +3 . For these two retinal locations, there was a substantial and nearly constant difference in $\overline{\mathrm{RT}}$ for Reading Positions 4 and 7 (see Figure 3). These results are convincing evidence of a reading position effect not accountable for by lateral interference effects associated with retinal location.

Probably the most interesting result of the present experiments is the additivity of memory set size and reading position. None of the current theories predicted this; and, to the extent that this result can be repeated and found under other conditions, it is 
a challenge requiring elaboration of the current theories. An experiment in progress is investigating the conditions under which this additivity obtains.

\section{REFERENCE NOTES}

1. Sternberg. S. Scanning a persisting visual image versus a memorized list. Paper presented at Eastern Psychological Association. Boston. Massachusetts, April 1967.

2. Sternberg. S. Existence and transformation of iconic memory revealed by search rates. Paper presented at the annual meeting of the Psychonomic Society, Boston. Massachusetts, November 1974.

\section{REFERENCES}

Eriksen. C. W. . \& Spencer. T. Rate of information processing in visual perception: Some results and methodological considerations. Journal of Experimental Psychology Monograph. 1969. 79 (No. 2. Part 2).

Estes. W. K. Interactions of signal and background variables in visual processing. Perception \& Psychophysics, 1972, 12. 278-286.

Estes. W. K. Redundancy of noise elements and signals in visual detection of letters. Perception \& Psychophysics. 1974, 16, 53-60.

Estes, W. K.. Allmeyer. D. H.. \& Reder. S. M. Serial position functions for letter identification at brief and extended exposure durations. Perception \& Psychophvsics, 1976, 19, 1-15.

Estes. W. K..\& Wolford, G. L. Effects of spaces on report from tach istoscopically presented letter strings. Psychonomic Science, 1971, 25. 77.80.

GARDNER. G. T. Evidence for independent parallel channels in tachistoscopic perception. Cognitive Psychology. 1973. 4. 130-155. (a)

Gardner. G. T. Parallel perceptual processing and decisional strategies: A reinterpretation of the Shaw and LaBerge effect. Perception \& Psychophysics. 1973, 13, 517-518. (b)

MC IntYre, C. . Fox, R., \& Neale, J. Effects of noise similarity and redundancy on the information processed from brief visual displays. Perception \& Psychophysics, 1970, 7, 238-332.

Mclaughlin, J. P., Masterson, F. A., \& Herrmann, D. J. Pattern redundancy and detection in very short-term memory. Perception \& Psychophysics, 1972, 12, 205-208.

Nickerson. R. S. Response times with a memory-dependent decision task. Journal of Experimental Psychology, 1966, 72, $761-769$.

RUMELHART, D. E. A multicomponent theory of the perception of briefly exposed visual arrays. Journal of Mathematical Psychology, 1970, 7. 191-218.

SHAW, P. Processing of tachistoscopic displays with controlled order of characters and spaces. Perception \& Psychophysics, 1969. 6. $257-266$.

Shaw, P.. \& LABerge, D. Manipulation of visual processing by varying the rewards associated with display locations. Perception \& Psychophysics, 1971, 10, 348-352.

TOW NSEND, J. T. A note on identifiability of parallel and serial processes. Perception \& Psychophysics, 1971, 10, 161-163.

Townsend, J. T., TAYloR, S. G., \& Brown, D. R. Lateral masking for letters with unlimited viewing time. Perception \& Psychophysics, 1971, 10, 375-378.

WINER. B. J. Statistical principles in experimental design. New York: McGraw-Hill. 1962.

Wolford, G. L.. \& Hollingsworth. S. Lateral masking in visual information processing. Perception \& Psychophysics, 1974, 16. 315-320. (a)

Wolford, G. L.. \& Hollingsworth, S. Retinal location and string position as important variables in visual information processing. Perception \& Psychophysics, 1974, 16, 316-320. (b)

(Received for publication December 12, 1975; revision accepted September 28, 1976.) 